ISSN: $1130-3743$

\title{
LA ANTROPOLOGÍA Y LA ETNOGRAFÍA EDUCATIVAS. APORTACIONES TEÓRICAS Y METODOLÓGICAS
}

\section{EDUCATIONAL ANTHROPOLOGY AND ETHNOGRAPHY. THEORETICAL AND METHODOLOGICAL CONTRIBUTIONS}

JOSÉ GONZÁLEZ MONTEAGUDO

Universidad de Sevilla. Facultad de Ciencias de la Educación. Departamento de Teoría e Historia de la Educación y Pedagogía Social. Avda. San Fco. Javier, s/n. 41005 Sevilla.

\section{RESUMEN}

Tradicionalmente se ha prestado poca atención a los enfoques antropológicos y etnográficos dentro del campo educativo. Sin embargo, en la actualidad estas temáticas provocan bastante atención y despiertan muchas expectativas. El objetivo de este trabajo consiste en presentar una visión panorámica y de conjunto de este campo.

En este artículo se analizan el origen y el desarrollo de la Antropología y etnografía educativas. Se presenta la evolución desde las perspectivas antropológicas clásicas a los enfoques más interpretativos y simbólicos. También sugerimos algunas de las direcciones emergentes que está tomando la etnografía educativa, tanto en Estados Unidos como en Gran Bretaña. Por último, mencionamos algunas de las publicaciones en lengua española más importantes en este ámbito. Pensamos que en el futuro habremos de dedicar más tiempo y energía para escribir e investigar en este importante campo.

\section{SUMMARY}

Traditionally it hasn't been paid enough attention to the anthropological and ethnographical approaches in the educational field. However, these matters are generating a lot of interest and expectation nowdays. The purpose of this work is to show a general view in this matter.

In this paper the origin and development of Educational Anthropology and Ethnography are analysed. The progress from classic anthropological perspectives 
to more interpretive and symbolic approaches is presented. We also suggest some emergent directions of educational ethnography in United States and Great Britain. Finally, some of the most outstanding spanish publications in this area are mentioned. We think that, in future, we should devote more time and energy to write and research into this important area.

La Antropología de la educación constituye un ámbito académico y de investigación que goza de una excelente salud en el momento presente. La ocupación y preocupación de un grupo cada vez más numeroso de antropólogos, pedagogos y sociólogos en relación con esta temática se ha visto reforzada por la inclusión como disciplina curricular de esta temática en diferentes titulaciones de las Facultades de Ciencias de la Educación. Pensamos que en este nuevo contexto se hace necesario que desde los ámbitos pedagógicos contribuyamos al desarrollo y a la consolidación institucional y académica de este campo del conocimiento. La importancia en las ciencias sociales de la perspectiva etnográfica y, más en general, de las aproximaciones cualitativas, es algo innegable. Junto a la Antropología y la Sociología (que cuentan con una fuerte tradición en estos asuntos), la Pedagogía y la Psicología parecen apostar en los últimos años por senderos teóricos y metodológicos de mayor creatividad (cf., por ejemplo, el caso de la Psicología Social en Sánchez-Candamio, 1995). Por nuestra parte, en este artículo nos limitaremos a presentar los enfoques antropológicos de orientación social y cultural. Esto no implica que consideremos poco adecuados los enfoques filosóficos. Unos y otros tienen objetivos diferentes, usan métodos distintos y ofrecen diversas posibilidades de cara al desarrollo del conocimiento educativo y a la intervención pedagógica (cf. Bouché et al., 1995).

1. El ENFOQue antropológico y la PERSPECTIVA ETNOGRÁfica dE LOS ESTUdios de CAMPO. DE LAS ANTROPOLOGÍAS HOLISTAS A LAS ANTROPOLOGÍAS INTERPRETATIVAS

La antropología, en el sentido en que aquí nos interesa (es decir, en tanto que antropología cultural), es el estudio de las conductas y de las sociedades humanas contemporáneas (Harrington, 1982, 132; como textos introductorios a la antropología, cf. Harris, 1983 y Jiménez Núñez, 1979; sobre la historia y las corrientes teóricas de la antropología, cf. Harris, 1968). La diferencia entre antropología y etnografía debe de ser aclarada al inicio de nuestra exposición. Mientras que aquélla es una disciplina científica, ésta alude al dispositivo metodológico con la que la primera trabaja. La investigación etnográfica consiste en la recolección y análisis de datos socioculturales de un grupo social o humano. También se suele denominar etnografía al producto final del proceso investigador, que consiste en un informe escrito en el cual se describe el sistema sociocultural analizado o bien algunos de sus componentes más importantes. Cuando se emprende una labor de comparación entre datos etnográficos pertenecientes a diferentes sistemas socioculturales tenemos que hablar de etnología. En el contexto de la lengua francesa, con frecuencia se identifican los términos etnología y etnografía (González Monteagudo, 1994, 72). 
El origen de la tradición de los estudios de campo podemos situarlo en Malinowski que fue el primer antropólogo que trató de fundamentar el método con el que trabajó en las islas de los Mares del Sur. Malinowski (1922) señaló que la etnografía, a diferencia de otras ciencias más exactas, no se ha ocupado de esclarecer sus métodos de trabajo, por lo que los autores "discurren sobre datos y conclusiones que surgen ante nuestros ojos sin la menor explicación" (Malinowski, 1922, 23). Reclama la necesidad de describir las circunstancias en que se efectuaron las observaciones y se recogió la información. También pide que se establezca una distinción clara entre las exposiciones e interpretaciones del indígena y las deducciones del investigador. El estudioso, dice nuestro autor, debe guiarse por criterios estrictamente científicos, convivir con los indígenas en un contacto tan estrecho como sea posible y utilizar métodos precisos en orden a recoger, manejar y establecer sus pruebas. Malinowski realiza una diferencia entre zambullirse esporádicamente en el medio de los indígenas y estar en auténtico contacto con ellos. "Los indígenas, al verme constantemente todos los días dejaron de interesarse, alarmarse o autocontrolarse por mi presencia, a la vez que yo dejé de ser un elemento disturbador en la vida tribal que me proponía estudiar" (Malinowski, 1922, 27). Malinowski rechaza las ideas preconcebidas que pueda tener el investigador, pero señala la importancia de las "conjeturas", que son "el don principal de un pensador científico" (Malinowski, 1922, 28). Es necesario, según Malinowski, tener en cuenta la mentalidad del indígena, es decir, sus concepciones, opiniones y formas de expresión. "La meta es, en resumen, llegar a captar el punto de vista del indígena, su posición ante la vida, comprender su visión de su mundo" (Malinowski, 1922, 41). El punto de vista de Malinowski, aunque envuelto en la mitología del trabajo de campo clásico (cf. Stocking, 1983), fue el primer intento de sentar las bases del enfoque etnográfico. Algunos de los dilemas que planteó este autor siguen siendo los dilemas que nos planteamos, en la actualidad, en los trabajos de campo.

Entre los años 1925 a 1954 un gran número de antropólogos investigó o escribió sobre los sistemas formalizados de educación y de enculturación de los niños. Entre otros, hay que mencionar a Bateson, Benedict, Evans-Pritchard, Herskovits, M. Mead, Radin, Redfield, Sapir, Whiting y Boas (Eddy, 1985, 84-92; García Castaño y Pulido, 1994, 25-39).

En este período cobró auge el enfoque de cultura y personalidad. Los antropólogos estudiaron las pautas de educación informal y trataron de relacionarlas con los rasgos de personalidad, comparando diferentes sociedades. Desde esta perspectiva, representada sobre todo por Benedict y por M. Mead, la cultura configura la personalidad. De ahí el término de configuracionismo, aplicable sobre todo a Benedict (retomaremos esta teoría al discutir, más adelante, el concepto de cultura). En este ámbito también hay que situar el enfoque de la personalidad como base mediadora de Kardiner (sobre cultura y personalidad, cf. García Castaño y Pulido, 1994, 28-35; Harris, 1968, 340-401; Harris, 1983, 499-547; Jiménez Núñez, 1979, 137-156; Janer y Colom, 1995, 83-85).

Aunque resulta muy problemático presentar en unas pocas líneas algunas de las tendencias que han marcado la evolución de la antropología (puesto que incluso en el caso de cada autor existe una evolución que supone un cambio de enfo- 
que o de modelo; por ejemplo, éste es el caso de M. Mead; cf. Mead, 1972, sobre sus experiencias personales y científicas), nos ha parecido conveniente ofrecer un breve apunte sobre esta cuestión. A juicio de Sanday (1983, 22-34), existen tres estilos paradigmáticos de etnografía. El primero es el estilo bolista. Aquí hemos de situar las orientaciones funcionalistas (Malinowski, Radcliffe-Brown) y configuracionistas (Benedict y M. Mead, ambas discípulas de Boas). El estilo holista, muy ligado en las últimas décadas al estructural-funcionalismo (dado que RadcliffeBrown integró el concepto de función procedente de Durkheim), concibe a la sociedad como un sistema compuesto de partes mutuamente dependientes. Algunos de los conceptos relevantes en el estilo holista son: proceso, mantenimiento, supervivencia, adaptación y cambio. El segundo estilo es el semiótico, animado por la búsqueda del punto de vista del nativo. En este estilo hemos de incluir la antropología interpretativa de Geertz, el cual enfatiza la capacidad de simbolización de las personas, y la antropología cognitiva o etnociencia de Goodenough, quien define la cultura en términos ideacionales y mentalistas. Sobre el estilo semiótico volveremos nuevamente, al comentar la influencia del planteamiento de Geertz sobre la antropología actual. El tercero de los enfoques es el estilo comportamentalista o conductista. En esta perspectiva lo importante es la conducta. Para estudiarla, se plantea la formulación de proposiciones de carácter deductivo mediante la reunión de datos observacionales sobre categorías funcionalmente relevantes preseleccionadas dirigidas al descubrimiento de la convariación de patrones en la conducta observada. Este enfoque está asociado al famoso estudio en seis culturas llevado a cabo, entre otros, por los Whiting. Para resumir estos tres estilos, Sanday $(1983,34)$ recuerda que el centro de interés de ellos es, respectivamente, la globalidad ("whole"), el significado y la conducta.

Según Zaharlick $(1992,117)$, la cultura es uno de los conceptos vertebradores de la antropología. La cultura alude al modo global de vida compartida por un grupo humano. Ello implica un mayor énfasis sobre el grupo respecto del individuo. La cultura es algo aprendido socialmente, que es objeto de transmisión y de comunicación. Más adelante comentaremos más detenidamente el concepto de cultura, pero como primera aproximación puede servirnos este concepto inicial.

El enfoque antropológico se caracteriza por (cf. García Castaño y Pulido, 1994, 8-24; Zaharlick, 1992, 117):

1. Intentar una comprensión holística, global y en profundidad de las actividades, comportamientos y valores bumanos, contemplados en el marco de su contexto social y cultural. Esta comprensión debe de incluir los fenómenos relativos a la transmisión y adquisición de cultura. Con este fin, los antropólogos suelen acudir a las dimensiones "macro" para poder entender los fenómenos "micro" de la realidad social.

2. Una actitud de relativismo cultural, en el doble sentido de estimar como válidos los modos de vida estudiados y, además, como enfoque metodológico para la descripción y comprensión de esos modos de vida.

3. Un marco comparativo, a través del cual los estudios realizados pueden contemplarse en una perspectiva transcultural. 
Desde la óptica antropológica, las características principales de la investigación etnográfica son las siguientes (Zaharlick, 1992, 119-121):

- Relaciones sociales. El etnógrafo y los sujetos crean un espacio de relación social de carácter difuso y prolongado, en diferentes contextos.

- El investigador como aprendiz. La relación entre el observador y los participantes no ha de ser asimétrica, sobre todo en las primeras etapas de la estancia el investigador ha de asumir un papel de "estudiante".

- Observación directa. No hay otro medio más que el de la observación directa para captar lo que Malinowski llamaba "los imponderables de la vida real" (cf. Malinowski, 1922, 36), es decir, los fenómenos que sólo puede aprehenderse en su plena realidad y no a través de entrevistas.

- Observación participante, naturalista y de largo plazo. Se trata de captar el flujo de la vida social, participando en las actividades cotidianas durante un período prolongado de tiempo.

- Enfoque ecléctico. Los etnógrafos usan técnicas de recogida y análisis de datos de muy diferente tipo. Las técnicas empleadas y el tipo de datos requeridos dependen de las características concretas de la investigación a realizar.

- El etnógrafo como instrumento de investigación. La persona entera del investigador participa en el proceso de investigación seleccionando de una manera personal y creativa la información más relevante y otorgándole una determinada significación en el contexto global del estudio en cuestión.

- Orientación interactiva-reactiva. La etnografía no es un proceso lineal y cerrado, "pre-visto" con antelación. Por el contrario, tiene un carácter interactivo, de modo que a lo largo de las diferentes fases del proceso se procede a la revisión de los objetivos, focos de atención y procedimientos establecidos en las fases iniciales de la investigación.

- Perspectiva bolística, bumanística y transcultural. En la etnografía existe una gran preocupación por el ámbito global del escenario social analizado, la problemática humana y vital de los sujetos con los que se convive y el establecimiento de marcos de análisis de carácter comparativo.

Como ya hemos indicado, el elemento central del análisis antropológico es el concepto de cultura (cf. Geertz, 1973, 1-59; Spradley, 1980, 3-16; Bogdan y Biklen, 1982, 35-37; Stenhouse, 1981, 31-52; Wolcott, 1985; Zaharlick, 1992; Janer y Colom, 1995; Colom y Mèlich, 1994; Rivas, 1992, 27-67). La de cultura es una noción tan polisémica y de un significado tan ambiguo y contradictorio que en muchas ocasiones resulta difícil comprender la utilidad del concepto.

La cultura es algo colectivamente transmitido, aprendido y compartido, según la tradición sociológica y antropológica en la línea de Parsons (cf. Stenhouse, 1981, 32). Para los antropólogos cognitivos y etnocientíficos, como por ejemplo Spradley $(1980,5)$, "La cultura es el conocimiento adquirido que la gente usa para interpretar la experiencia y generar la conducta sociab. Otros antropólogos, como Wax, insisten en resaltar el carácter de significado compartido que posee la cultura. Para Geertz (1973, 1-59) y los antropólogos interpretativistas, la cultura es un contexto densamente descrito y un sistema interconectado de signos interpreta- 
bles (cf. Bogdan y Biklen, 1982, 35-37). Stenhouse (1981, 33-34) se sitúa en una línea parecida al afirmar que la cultura es el medio a través del cual interactúan las mentes humanas en comunicación. Para este pedagogo, la cultura, al mismo tiempo que es un producto de la acción social, es también un determinante de la misma.

Las definiciones de cultura son múltiples. Rivas (1992, 36-47) cita las de Orozco, Coll, Tylor (el antropólogo del siglo XIX creador del concepto clásico y omniabarcador de la cultura), Rossi y O'Higgins, Sapir, Aguirre, Willis y Bourdieu. Después de este repaso, Rivas añade su propia definición a la lista anterior, sintetizando y unificando los diferentes matices del concepto:

La cultura es un producto de la interacción social; que actúa como mecanismo regulador de la complejidad que supone y como adaptación del individuo y del grupo al ecosistema en que se encuentra; como forma de solucionar sus problemas básicos; que se compone de modos de vida y formas de pensamiento; aprendidos e interiorizados por los sujetos que la forman, en forma de herencia cultural que se transmite; y mediante la cual es posible el progreso y el cambio de la propia cultura y del grupo en el que se desarrolla (Rivas, 1992, 47).

Rivas $(1992,37)$ recuerda que, básicamente, hay dos grandes orientaciones en la conceptualización de la cultura: la orientación materialista resalta la importancia de las formas de vida y de la praxis social y productiva (sobre esta corriente, cf. Harris, 1968, 549-596); la orientación idealista piensa la cultura sobre todo en términos de formas de pensamiento.

El principal problema que plantea el concepto de cultura consiste en la rigidez con que se ha concebido y usado en buena parte de los enfoques antropológicos. Entre las propiedades de la cultura, por lo menos en su concepción holista, que ha sido la más influyente, se suelen mencionar la estabilidad (es algo relativamente permanente en el tiempo), la predictibilidad (es un sistema de expectativas, que permite conocer de antemano los tipos de conducta admitidos, prescritos o castigados) y la conformación de la identidad del grupo y de los individuos que lo componen (en base a unas formas de vida y de pensamiento comunes) (cf. Rivas, 1992, 47-50). Desde este enfoque holista, es posible analizar los patrones, pautas o modelos ("patterns") que configuran la vida de las personas. En esta línea se sitúa Benedict (cf. Sanday, 1983, 24), al hablar de la tendencia hacia la consistencia en el sentido de que cada cultura selecciona, de entre la infinita variedad de posibilidades conductuales, un segmento limitado que en ocasiones se constituye en una configuración. Las configuraciones pautan la existencia y condicionan las reacciones emotivas y cognitivas de los sujetos.

Este concepto de cultura es poco útil para dar cuenta del pluralismo y de la diversidad culturales, sobre todo en el ámbito tan variado y cambiante de las sociedades occidentales contemporáneas. Por eso es necesario ampliar el concepto de cultura. Según Stenhouse $(1981,32)$, nuestra sociedad pluralista contiene en su seno tradiciones múltiples, diferentes y con frecuencia lógicamente incompatibles. A juicio de este autor, el término "subculturas" no soluciona el problema de la diversidad cultural, puesto que sugiere una jerarquía de culturas de diferente 
rango. En la actualidad, sin embargo, el término subculturas es de amplio uso en las ciencias sociales y los investigadores señalan que posee un valor descriptivo y no valorativo (cf. Fernández Enguita, 1990). Otros autores hablan de "microculturas" (Wolcott, 1985, 133) y de "nivel cultural" (Willis, 1977, 216) para hacer un uso más contextualizado y dinámico de la cultura. Por su parte, Janer, y Colom (1995, 72-78), junto a los enfoques clásicos del concepto de cultura, resaltan la importancia de la cultura popular y de masas y de las culturas de clase social.

La cultura se compone de los siguientes elementos: contenidos, símbolos y modelos comunicativos (en este punto seguimos a Rivas, 1992, 56-67, que revisa estos elementos y los sitúa en el ámbito escolar). El contenido se refiere a las creencias y a los valores. Los simbolos son los soportes de la comunicación y del intercambio social; cumplen funciones de comunicación (y aquí encontramos la compleja relación entre pensamiento y lenguaje, tan discutida en la psicología, sociología y antropología contemporáneas) y de participación (símbolos de solidaridad, de organización jerárquica, del pasado tradicional, míticos y religiosos, políticos, etc.). La relación entre creencias y valores, por un lado, y símbolos, por otro, se produce mediante determinados procesos que, al regular las relaciones y el intercambio entre los sujetos, establecen los ámbitos de la actuación cultural. Aquí hemos de situar los procesos de comunicación y los rituales y ceremonias. Según Rivas (1992, 62-67), el rito es la expresión de la forma de pensar y de sentir de una comunidad. El rito introduce a los sujetos en las formas tradicionales de la cultura y les prepara para el futuro cultural. En definitiva, hay que considerar el ritual como:

...marco para la expresión y participación del individuo en su cultura, así como para su transmisión. Esto es, entendemos el ritual como una manifestación cultural a través de la cual los sujetos, en especial los niños sometidos fundamentalmente a procesos de socialización, aprenden a simbolizar el mundo y aprenden cómo ordenar la vida en ese universo de significados. En función de esto, los individuos tomarán parte en múltiples rituales, en múltiples niveles de participación y de lealtad, del grupo social (Rivas, 1992, 63).

El modelo cultural que se desprende de esta perspectiva es un modelo de la vida social sumamente pautado, con rituales precisos y con un alto poder regulador de los actores sociales.

En las dos últimas décadas se ha producido un fuerte cuestionamiento del estilo holista. A partir sobre todo de la publicación de una obra recopilatoria por parte de Geertz (1973; esta obra abarca textos escritos desde 1957 a 1972. Cf. también Geertz, 1988; Geertz et al., 1992; Schwandt, 1994, 122-123; Díaz Viana, 1991), el estilo semiótico ha cobrado fuerza en el panorama antropológico. El título del libro, La interpretación de las culturas, es muy elocuente. Geertz sigue sirviéndose del concepto clave de la antropología, pero quiere imprimir en ésta un "giro interpretativo". Este giro, como el "giro lingüístico" propiciado algunos años antes por Rorty, ha tenido una decisiva influencia en las ciencias sociales en su conjunto. Geertz se opone a la perspectiva del análisis cultural derivada del estructura- 
lismo y, en especial, de la antropología cognitiva o etnociencia. Desde el punto de vista estructuralista, las categorías y estructuras de la cultura proporcionan mecanismos altamente explicatorios sobre las conductas de los miembros de una sociedad dada. Según Geertz, se trata de un enfoque reduccionista, puesto que pretende imponer una verdad científica, aunque, de hecho, sólo se trata de una determinada interpretación de la cultura, pero no la única posible. También rechaza la metodología estructuralista, dado que reifica el mundo de la experiencia vivida transformándolo en un lenguaje científico especializado (cf. Schwandt, 1994, 122). Según Reynoso (en la "Introducción" a Geertz, 1973, 9), Geertz propugna "una lectura del quehacer humano como texto y de la acción simbólica como drama, reivindicando la capacidad expresiva de una retórica autoconsciente".

Esta actitud problematizadora en torno a la cultura se percibe también en los llamados estudios culturales, que se proponen un acercamiento no fragmentario y no reduccionista a la cultura, entendida como proyecto político de crítica y transformación social (Giroux, 1988, 193-208).

Geertz (1973, 19-20) se propone reducir el concepto de cultura a sus verdaderas dimensiones y sacarlo del pantano conceptual en que lo ha convertido el estilo tyloriano tan arraigado en la tradición antropológica. "Lo que nosotros llamamos nuestros datos - escribe-son realmente interpretaciones de interpretaciones de otras personas sobre lo que ellas y sus compatriotas piensan y sienten" (Geertz, 1973, 23). Para Geertz (1973, 24), el debate sobre si la cultura es objetiva o subjetiva está mal planteado (de paso, recuerda la inutilidad de los insultos intelectuales que este debate acarrea: “idealista!”, «imentalista!”, “iconductista!”, «impresionista!", "ipositivista!»). Al concebir la conducta humana como acción simbólica carece de sentido la discusión ontológica. Por lo que hay que preguntarse es por el sentido y el valor de esa conducta. Este enfoque acerca de Geertz a las perspectivas que enfatizan el punto de vista del actor, el análisis «emic"y la "Verstehen". "Todo el quid de un enfoque semiótico de la cultura es - dice Geertz- ayudarnos a lograr acceso al mundo conceptual en el que viven nuestros sujetos, de suerte que podamos, en el sentido amplio del término, conversar con ellos" (Geertz, 1973, 35).

La descripción etnográfica es interpretativa y densa (Geertz toma el concepto de "descripción densa" de Ryle); lo que interpreta es el flujo del discurso social; la interpretación "consiste en tratar de rescatar 'lo dicho' en ese discurso de sus ocasiones perecederas y fijarlo en términos susceptibles de consulta" (Geertz, 1973, 21 y 32). La descripción etnográfica debe moverse en un nivel microscópico, puesto que las interpretaciones más amplias o de gran escala deben realizarse partiendo de los abundantes conocimientos que podemos tener de cuestiones extremadamente pequeñas. La teoría cultural no es predictiva, pero puede producir una generalización parecida a lo que en medicina y en psicología profunda se llama inferencia clínica (Geertz, 1973, 36-37).

En una obra posterior, que ya refleja la sensibilidad postmoderna y deconstructiva de la década de los ochenta, Geertz (1988) propuso un análisis de la antropología como obra literaria, estudiando cuatro "estilos" diferentes en las obras de Levi-Strauss, Evans-Pritchard, Malinowski y Benedict. Como escribe Díaz Viana 
(1991, 15), este libro de Geertz nos conduce a la visión de la etnografía como una verdadera poética de la persuasión en la que importa menos la amplitud de las descripciones y de los datos que la habilidad del etnógrafo para convencernos de que es verdad lo que cuenta. La importancia de la importancia de la llamada Antropología simbólica (etiqueta bajo la cual podemos agrupar a una gran variedad de corrientes actuales, y no sólo a Geertz) para una reconsideración creativa y antipositivista de la acción educativa ha sido mostrada claramente por Mèlich (1996) en un interesante libro de reciente aparición.

Inspirándose en Geertz, un numeroso grupo de antropólogos se han propuesto, a partir de mediados de la década de los ochenta, acabar con lo que llaman algunos lugares comunes de la antropología cultural y revitalizar la discusión mediante un intento de reinterpretación de la escritura etnográfica (cf. Clifford, 1986; Tyler, 1986; Marcus, 1986; Marcus, 1994; Clifford y Marcus, 1986; Díaz Viana, 1991).

Clifford (1986, 25-60), en la Introducción a uno de los textos de esta corriente, dice que en la etnografía no hay verdades absolutas, sino parciales e incompletas. Defiende un método dialogal (se remite en este punto a Bakhtin) con espacio discursivo propio de la etnografía. Clifford analiza el cuestionamiento del sujeto investigador en el proceso de investigación, recordando que algunos autores seguidores de la Escuela de Chicago (como W. F. Whyte) asumieron el elemento subjetivo implícito en la postura del investigador, trasladando sus reflexiones personales a los informes etnográficos. De igual manera, Clifford comenta la crisis provocada en la sociología convencional por las posiciones etnometodológicas, cuestionadoras del razonamiento sociológico profesional. Apoyándose en estas corrientes, Clifford concluye su texto resaltando la importancia de lo poético $y$ de la escritura:

Sólo gracias al auge de la semiótica, del post-estructuralismo y de la hermenéutica, podemos hablar con propiedad acerca de la aproximación a la realidad, de una praxis realista. ¡Pero dejemos al realismo para otra ocasión! Reconozcamos, mejor, las dimensiones poéticas de la etnografía, para lo cual no hay sino que desprejuiciarse. Lo poético no es cosa circunscrita a una visión romántica, modernista o subjetiva; también puede ser lo histórico, lo preciso, lo objetivo... Y, naturalmente, lo mismo acontece con eso que llamamos prosa. La etnografía debe ser hiperescritura, actividad textual: trasvase continuo de géneros y de disciplinas" (Cliffrod, 1986, 59-60).

\section{La antropología de la EDUCACión y la etNografía educativa en los Estados} UNIDOS

La antropología de la educación como subdisciplina académica en los Estados Unidos ha tenido una implantación progresiva (sobre la evolución histórica y el estado actual de la subdisciplina, cf. Eddy, 1985; Sirota, 1987, 78-81; Ogbu, 1989, 291-294; Schensul, 1985; García Castaño y Pulido, 1994, 25-39 y 45-54).

El momento clave para el inicio de este proceso parece ser el año 1954. En esta fecha, Spindler, convocó en Stanford la famosa Conferencia sobre educación 
y antropología, cuyas actas editó él mismo al año siguiente. Este período se caracterizó por un aumento de los recursos económicos disponibles para la investigación en antropología, el desarrollo de un enfoque interdisciplinar (iniciado antes de la Segunda Guerra Mundial, con constitución de departamentos conjuntos de sociología y antropología) y una notable expansión académica de la antropología dentro de las universidades (cf. Eddy, 1985, 84-92).

Los primeros antropólogos educativos se inspiraron en el trabajo desarrollado por los pioneros del estudio de la socialización, algunos de los cuales ya fueron mencionados en el epígrafe anterior. En los años sesenta aumentó el número de estudiantes de antropología, surgió un fuerte interés hacia la antropología aplicada (incluyendo el estudio de ambientes educativos no escolares) y se produjo un proceso de especialización dentro del área. El alto interés hacia la antropología de la educación se explica por la crisis social y política vivida en los años sesenta (derechos civiles; integración de las minorías; etc.). Por otra parte, en 1962, Bruner comenzó a dirigir los trabajos sobre el programa curricular de orientación antropológica Man: A course of study (cf. Torres, 1994, 213-220, para una descripción de este programa). Este hecho representa el intento de incluir la enseñanza de la antropología dentro del curriculum escolar. Otra de las tareas que emprendieron los antropólogos fue contrarrestar los errores implícitos en el concepto de cultura manejado por los psicólogos, los cuales resaltaron el modelo del déficit y de la deprivación, atribuyendo a variables psicológicas y personales el fracaso escolar de los niños negros y de otras minorías. Los antropólogos negaron estas interpretaciones y resaltaron la importancia del contexto cultural y de las discontinuidades culturales en la explicación del fracaso escolar. En esta época se publicaron colecciones de libros sobre antropología y G. y L. Spindler alentaron la realización y publicación de numerosas investigaciones, tanto en Alemania como en Estado Unidos.

Este auge culminó en 1970 con la creación del Council on Anthropology $\&$ Education, que coordinó la actividad profesional e investigadora del creciente número de antropólogos orientados hacia la educación. El "Council» creó un boletín, que se transformó en revista en 1977; se trata de Antbropolo gy E Education Quarterly, influyente publicación en ámbitos de la psicología, la sociología, la antropología y la pedagogía. La subdisciplina comenzó su andadura muy influida por los modelos de cultura y personalidad. Uno de los retos que se plantearon los antropólogos educativos fue corregir la orientación de la investigación convencional sobre educación de aquella época. Esta investigación, a juicio de los antropólogos, resultaba artificial, era confusa y descuidaba el contexto natural del comportamiento (cf. Ogbu, 1989, 291-294; Eddy, 1985, 92-98).

A mediados de la década de los ochenta, Schensul $(1985,63-68)$, que fue presidente del "Council" realizaba un balance de la situación de la subdisciplina. Existía en ese momento un estancamiento del desarrollo de la antropología de la educación, tanto en cuanto a extensión académica como a número de estudiantes. El ámbito de la antropología se extendió desde las universidades a las escuelas, las comunidades locales y otros ambientes de socialización. Los antropólogos de los años ochenta apostaban por el pluralismo cultural y se preocupaban por 
estudiar y superar las discontinuidades entre las escuelas y las comunidades locales. La orientación de los estudios antropológicos tendía a ser, y esto es lógico por el clima intelectual de la pasada década, más fenomenológica, multimodal, interpretativa y naturalista. Schensul concluye su balance afirmando que ya no es preciso que los antropólogos separen su "trabajo teórico" de su "intervención como ciudadanos".

Los principales marcos analíticos empleados en antropología de la educación han sido, según Ogbu (1989, 297, 302), el modelo instrumental de Spindler (más adelante lo comentaremos), los modelos transaccionales de Gearing (sobre este autor, cf. García Castaño y Pulido, 1994, 55-66) y los modelos interactivos-interpretativos. En estos últimos, Ogbu incluye el interaccionismo simbólico, la etnometodología, la sociolingüística, el funcionalismo estructural y el enfoque ecológico. Por su parte, Harrington $(1982,135-136)$ menciona las siguientes perspectivas teóricas en la antropología reciente: estructural-funcionalismo, teoría neoweberiana, interaccionismo simbólico y etnometodología, fenomenología, estructuralismo, etnociencia, etología y teoría marxista.

Las aportaciones más significativas de la etnografía a la educación son una contribución a la mejora de la educación y una visión amplia, contextualizada culturalmente y de nivel múltiple de la educación, entendida en un marco más amplio que la mera escolarización. Los enfoques dirigidos a la mejora de la instrucción, del curriculum y de la evaluación, según el punto de vista antropológico, deben estar orientados por el conocimiento y la comprensión del contexto global que rodea la escolarización, si no quieren verse abocados a un probable fracaso. El análisis antropológico de las discontinuidades entre la escuela y la familia y, más en general, entre la enseñanza y los elementos socioculturales, sugiere modos alternativos de organizar la enseñanza para que responda mejor al medio social (cf. Zaharlick, 1992, 121-123; Harrington, 134-155; Ogbu, 1981; Wolcott, 1985).

Entre las principales revisiones de la subdisciplina figuran las de Ogbu (1989) y Wilcox (1982 a). Reflexiones y propuestas de gran interés sobre la etnografía educativa pueden consultarse en Hymes (1982), Wolcott (1985) y Ogbu (1981).

La figura que ha protagonizado el desarrollo de la antropología de la educación ha sido G. Spindler (cf. Spindler, 1982, Spindler, 1987; Spindler y Spindler, 1987 a; Spindler y Spindler, 1987 b; Spindler y Spindler, 1992; sobre este autor, cf. García Castaño y Pulido, 1994, 45-54). Spindler, situado en la etnografia bolista, ha propuesto diferentes modelos para explicar la transmisión cultural. Uno de ellos es el modelo instrumental, aplicado en la investigación de campo mediante el Inventario de actividades instrumentales (IAI). Spindler trató de explicar por qué se eligen determinados comportamientos de entre la amplia variedad de posibilidades que existen. Mediante la técnica del IAI (que consta de una serie de dibujos que, en una línea parecida, al menos en parte, a las técnicas proyectivas estandarizadas, representan escenas de la vida social del grupo social estudiado), los Spindler aplicaron el modelo instrumental en el estudio de la comunidad alemana de Schönhausen, que vivía un acelerado proceso de cambio social y de modernización. el objetivo era comprender las relaciones entre cómo la gente per- 
cibe y responde al mundo que le rodea y sus adaptaciones socioculturales manifiestas. Spindler se preguuntó por el papel de la escuela como agente transmisor de cultura en un medio cambiante como el de Schönhausen. Spindler comprobó que la escuela transmitía una visión más tradicional y rural que moderna y urbana, idealizando aspectos como la tierra, el pueblo y los oficios tradicionales. En cambio, entre las preferencias instrumentales de las gentes del pueblo figuraban el confort, ingresos económicos regulares y horas fijas de trabajo, es decir, un modelo más urbano. En el curso de estas investigaciones, que incluían observaciones, administración de pruebas, análisis de documentos y entrevistas, los Spindler idearon la entrevista autobiográfica expresiva, que es una mezcla de entrevista expresiva estructurada y autobiografía cronológica abreviada. El enfoque de estos autores incluye tanto técnicas cuantitativas como cualitativas (cf. García Castaño y Pulido, 1994, 45-54; Spindler, 1987 b, 25-27). Los Spindler han estudiado en diferentes contextos culturales y geográficos el papel de las escuelas en sociedades cambiantes. En un estudio que recopila información procedente de culturas diferentes, Spindler $(1987,233)$ pone de relieve que las principales funciones de los sitemas culturales son el reclutamiento y el mantenimiento. En estos contextos, las escuelas implantadas con un objetivo modernizador producen una fuerte discontinuidad cultural, puesto que dejan de preparar a los niños para los roles sociales tradicionales. Esta es una de las causas de que lo que se enseña tienda a convertirse en algo formalizado y al margen de la realidad de los niños (Spindler, 1987, 236).

Otro autor pionero de la antropología de la educación y colaborador de Spindler en los años cincuenta fue Kimball (1955). Este antropólogo defendió, en una perspectiva igualmente holista, la aplicación del método de la historia natural (extraído de la ecología biológica) a los problemas de la transmisión y adquisición de cultura. Según Kimball $(1955,12)$, el método de la historia natural pretende la búsqueda de relaciones significativas entre los diferentes componentes presentes en una situación dada.

Entre los discípulos de Spindler hemos de destacar a Wolcott (cf. Wolcott, 1974; Wolcott, 1982; Wolcott, 1985; Wolcott, 1992; Wolcott, 1994; sobre este autor, cf. García Castaño y Pulido, 1994, 78-83). Wolcott escribió The Man in the Principal's office: An Ethnography, una de las etnografías educativas más célebres (cf. Wolcott, 1994, 115-148). Wolcott acompañó durante dos años a un director de escuela, dentro y fuera del centro escolar. Algunas de las conclusiones a las que llegó son la carencia de conocimiento profesional asociado con el rol de director y la proclividad hacia la conducta de "reducción de la variedad", para hacer más manejable la realidad cotidiana (Wolcott, 1994, 115-149; la fecha original de este texto es 1974). Con anterioridad, realizó trabajos de campo en un poblado de los indios Kwakiutl, en donde ejerció durante un año como maestro de una escuela unitaria. De esa investigación surgió una interesante reflexión sobre el papel del maestro como "enemigo" y sobre las dificultades de su tarea en un contexto transcultural (Wolcott, 1974). Wolcott también se ha ocupado de los modelos de adquisición de cultura (cf. García Castaño y Pulido, 1994, 78-83) y de los requisitos que deben cumplir las etnografias educativas. Wolcott (1985, 130 ss.) considera que el 
propósito de la investigación etnográfica es la descripción e interpretación del comportamiento cultural. Desde esta perspectiva, considera que la mayor parte de la llamada etnografía escolar consiste en la unión de investigación descriptiva con los esfuerzos por la mejora y el cambio educativos. "No se puede llegar -escribe- $\mathrm{a}$ un entendimiento equilibrado o completo de lo que los educadores están haciendo o de cómo van a actuar, si las dimensiones culturales del comportamiento humano se ignoran u oscurecen.... (Wolcott, 1985, 142).

Entre los etnógrafos recientes, llaman la atención los estudios de Ogbu (1974; 1981; 1989), que ha desarrollado su trabajo en los Estados Unidos, aunque es de origen nigeriano. En el primero de los textos citados, Ogbu llevó a cabo una investigación etnográfica de nivel múltiple, porque tenía como objetivo explicar en diferentes niveles el "fracaso" escolar y social de las minorías negras de una ciudad norteamericana. En este estudio analiza el papel de los profesores en el mantenimiento del fracaso escolar (cf. Ogbu, 1974, 133-169). Para dar cuenta de los múltiples niveles que operan en la realidad. Ogbu (1981, 161-163) propone una etnoecología de la escolarización, que tendría como objetivo el estudio de las relaciones entre estos tres elementos: la estructura ecológica (el contexto social y económico), la estructura cognitiva (los sistemas de percepción y de interpretación de los sujetos) y la conducta escolar efectiva. Según Charlot, Ogbu es uno de los pocos antropólogos de la educación norteamericanos orientado por una problemática más "ecológica" que estructural-funcional (Charlot, 1989, 105).

\section{LA ETNOGRAFÍA INTERACCIONISTA Y NEOMARXISTA EN GRAN BRETAÑA}

La tradición etnográfica británica es muy reciente (data de finales de los sesenta) y está vinculada sobre todo con el ámbito disciplinar de la sociología. En el Reino Unido, el concepto de cultura ha tenido muy escaso arraigo. En cambio, los investigadores británicos suelen usar el término "sociedad", lo que implica una preocupación por las desigualdades sociales y por los factores de diferenciación de clase social. Mientras que la etnografía inglesa piensa más en términos de interacción, la etnografía americana opera más en términos de comunicación y de transmisión cultural (cf. Woods, 1990 d; Charlot, 1989; Sirota, 1987; Atkinson et al., 1988). En la comparación realizada por Atkinson y Delamont (1990, 111-125) entre el estudio de los profesores en las tradiciones etnográficas inglesa y norteamericana, estos autores concluyen que los investigadores británicos realizan más citas directas de registros verbales de los docentes y que los profesores no sólo "hablan" en los estudios, sino que tienen una "identidad". En cambio, en la etnografía americana son descritos en términos de categorías. Estas diferencias explicarían la particular orientación de la etnografía inglesa, tanto en su versión interaccionista, la más importante, como en su versión neomarxista, que ha experimentado un fuerte desarrollo en los últimos años.

El enfoque ligado al interaccionismo simbólico ha sido difundido sobre todo por Woods, catedrático muy vinculado a la práctica educativa (fue profesor de primaria y de secundaria y en el ámbito universitario se ha ocupado de la formación 
pemanente de los profesores). La obra de Woods es abundante (cf. Woods, 1986; 1990 a; 1990 b; 1990 c; 1990 d; 1992; 1994; Woods y Hammersley, 1994. Sobre Woods, cf. Lapassade, 1991, 123-141; Charlot, 1989; Berthier y Legrand, Introducción a Woods, 1990 b, 7-24; Sirota, 1987, 75-78).

Junto a Woods, otros autores que podemos situar dentro del enfoque interaccionista son Hammersley (cf. Hammersley, 1984 a; 1984 b; 1990; 1992; Hammersley y Atkinson, 1983, recientemente traducido al español), Hargreaves (1992), Delamont (1976), Delamont y Hamilton (1978) y Delamont y Galton (1986).

Sin embargo, los primeros en abrir la "caja negra" de la escuela fueron Lacey y Hargreaves, a finales de los años sesenta, en fechas muy similares a los trabajos de Jackson (1968) y de otros autores norteamericanos, como Smith y Geoffrey (1968). Algunos años más tarde, Delamont y Hamilton (1978) realizaron un planteamiento crítico de los enfoques convencionales de investigación educativa y reclamaron un nuevo planteamiento.

En Woods encontramos según manifiesta él mismo en una entrevista, las influencias de la Escuela de Chicago y del interaccionismo simbólico. De todas formas, este autor se muestra contrario a la supuesta oposición paradigmática y considera que los métodos cualitativos no tienen por qué ser incompatibles con los cuantitativos (Woods, 1990 a).

Entre las publicaciones más importantes de Woods destacamos el libro escrito como introducción a la etnografía escolar (Woods, 1986) y que era, que sepamos, el único vertido al español, hasta la muy reciente traducción de una compilación de textos en colaboración con Hammersley (cf. Woods y Hammersley, 1994). En los últimos años, su escrito de mayor calado teórico y metodológico es el extenso capítulo sobre el interaccionismo simbólico que redactó para uno de los manuales norteamericanos sobre investigación cualitativa (cf. Woods, 1992, 338-404). En este texto, Woods analiza la evolución, los principios teóricos, las implicaciones metodológicas, el rol del investigador y la generación de la teoría desde la perspectiva del interaccionismo simbólico. Entre las implicaciones metodológicas menciona éstas: el respeto por el mundo empírico tal y como se da en la vida social cotidiana; el análisis de las diferentes capas de la realidad y de sus mutuas relaciones; la necesidad de asumir el rol del otro; la apreciación por la cultura y por las formas culturales peculiares; el aprendizaje de los símbolos del grupo estudiado, en particular del lenguaje y del vocabulario específico; la conveniencia de situar y contextualizar la interacción en los marcos en que se lleva a cabo; la comprensión de la interacción en términos de "flujo social", como proceso dinámico; la sensibilidad hacia la expresión del sí mismo ("self") en tanto que proceso; y, finalmente, la ubicación de la interacción simbólica en el ámbito social, puesto que el interaccionismo no es situacionista, ni idealista, ni empirista (Woods, 1992, 348-369).

Woods ha retomado y ampliado los conceptos clásicos del interaccionismo, aplicándolos a la vida cotidiana de los centros escolares.

El interaccionismo simbólico - dice Woods- mantiene que los seres humanos actúan hacia las cosas sobre la base del significado que las cosas tienen para ellos. La atribución de significados a los objetos es un proceso continuo que se lleva a 
cabo en contextos sociales. De esta manera, los interaccionistas simbólicos se centran sobre las perspectivas, por medio de las cuales la gente crea un sentido del mundo; las estrategias que la gente emplea para conseguir los fines que se propone; los diferentes contextos y situaciones en los cuales las personas definen sus fines; las culturas de los grupos en que interactúan; y, finalmente, sus carreras ["careers"] subjetivas, en tanto que opuestas a objetivas" (Woods, $1990 \mathrm{~d}$, 90).

Lapassade (1991, 143-151) ha resumido el enfoque de los etnógrafos interaccionistas británicos, centrado en el estudio de las perspectivas de los maestros y de los alumnos. Uno de los temas más estudiados ha sido el de las concepciones tradicionales de los docentes respecto de los alumnos: ¿cómo definen sus tareas los maestros?, ¿cómo ven a sus alumnos?, ¿cómo diferencian los "buenos" de los "malos" estudiantes? En el proceso de "etiquetaje", se produce una progresiva tipificación en tres fases: una inicial de especulación, otra intermedia de elaboración y la tercera de definición en términos de etiqueta. El análisis de la cultura de los maestros ha llevado a los etnógrafos a estudiar la ideología, el poder (el cual suele prevalecer sobre las convicciones pedagógicas) y la gestión del rol. Woods, Hargreaves y otros etnógrafos británicos han estudiado las perspectivas de los alumnos, categorizando a los estudiantes en función de las diferentes actitudes (de rechazo, aceptación conformista o defensa) sobre la escuela. El conjunto de estas investigaciones continúa sin ser traducido al español (cf. Woods y Hammersley, 1994).

En un artículo de 1985 (recogido en Woods, 1990 b, 144-169), Woods reflexionó sobre la utilidad de la sociología y de la etnografía para la práctica pedagógica. La sociología, dice Woods, no ha servido a los enseñantes; su abstracción teórica y su terminología especializada han alejado a los educadores. Lo mismo ocurre con la investigación realizada desde una óptica pedagógica. Para remediar esta situación, Woods propone realizar una investigación dirigida a los maestros mismos y no a los colegas sociólogos o pedagogos. La sociología debería mostrar a los estudiantes del ámbito pedagógico que la educación es una actividad social, que la enseñanza y el aprendizaje tienen lugar en circunstancias sociales y que la personalidad y el comportamiento son, al menos en parte, productos sociales. A partir de aquí, Woods analiza las posibilidades que la etnografía interaccionista tiene para comprender y mejorar la práctica educativa, por un lado, y para contribuir a una formación inicial y permanente de mayor calidad.

En uno de sus últimos artículos publicados, Woods (1994) estudia las estrategias de resistencia y de oposición creativa de los maestros británicos en el contexto del auge de la centralización y de la desprofesionalización docente propiciados por la reciente reforma educativa.

Antes hemos nombrado a Hammersley, autor de una importante obra etnográfica. Este investigador ha criticado el "naturalismo" empirista de muchos enfoques etnográficos y ha reclamado una etnografía caracterizada por el principio de reflexividad. A su juicio, la reflexividad tiene tres implicaciones: a) las acciones del investigador están abiertas al análisis y al debate críticos, en los mismos términos que las acciones de los demás participantes; b) el investigador debe hacerse cons- 
ciente de sus decisiones y de las motivaciones que subyacen a esas decisiones; y c) las actividades del investigador han de ser incluidas en el informe de investigación (Hammersley, 1984 b, 3-5).

Uno de los programas de investigación etnográfica más importantes llevados a cabo en el Reino Unido ha sido el proyecto "ORACLE". En el marco de este programa, Delamont y Galton (1986) publicaron una interesante y completa monografía sobre la escuela secundaria renovada. Las referencias que hemos ofrecido hasta aquí no han sido una idea muy limitda del enorme volumen de trabajo etnográfico realizado en los últimos treinta años en Inglaterra.

La etnografia neomarxista y crítica (cf. Willis, 1977; Willis, 1981; Feito, 1990; Everhart, 1983; Quantz, 1992; Carspecken y Apple, 1992; Roman, 1992; Roman y Apple, 1991) ha sido desarrollada, por lo que respecta a Inglaterra, a partir del conocido estudio llevado a cabo por Willis (1977, sobre Willis, cf. Feito, 1990, 5458 y Marcus, 1986, 245-262) sobre un grupo de doce "colegas" (así se llaman a sí mismos, en oposición a los estudiantes conformistas, los "pringaos") de un centro británico de secundaria, en la ciudad de "Hammertown". Willis observó a los chavales en sus reuniones informales, en las aulas y en otros lugares y entrevistó a sus padres y profesores. El tipo de etnografía propugnado por Willis pretende una consideración atenta del medio social del alumno. En este sentido, constituye el polo opuesto al enfoque de Woods, Furlong y otros etnógrafos de la interacción "cara a cara" (cf. Charlot, 1989, 99). Willis dividió su libro en dos partes: la primera es una "etnografia", de carácter descriptivo; y la segunda es un "análisis", de carácter teórico y sociológico, orientado por una perspectiva marxista dinámica y no reificada. Los elementos de la cultura oposicional obrera son la oposición a la autoridad y el rechazo del estudiante conformista (el "pringao"), la actitud racista y la conducta antiacadémica en el instituto ("Durmiéndose, escaqueándose y cachodeándose"; éste es el título de uno de los apartados del libro). En la descripción de la actividad educativa institucional, Willis resalta que la enseñanza funciona bajo una perspectiva de control y que los estudiantes tienen siempre una posición subordinada. Willis muestra las formas culturales propias creadas por los "colegas" como respuesta creativa ante su situación familiar, social y laboral (el estudio pretendía analizar la incorporación al mundo del trabajo de los adolescentes estudiados). En la parte final de la obra (Willis, 1977, 212-222), Willis analiza el nivel cultural como uuna entidad relativamente discreta con sus propias lógicas y su propia forma de penetración - distorsionada- de las condiciones reales de los agentes sociales implicados" (Willis, 1977, 216), situándose más allá de las teorías de la reproducción. Para Willis, la producción cultural, como dice uno de sus escritos, no es lo mismo que la reproducción cultural (cf. Willis, 1981).

Según Feito (1990, 22-23 y 54-58), la etnografia estructural de Willis pretende realizar una síntesis entre el enfoque interaccionista y las teorías de la reproducción (pues los análisis de Althusser, Bourdieu y B.Bernstein son parciales, porque desprecian la problemática del cambio social y porque tienen una imagen pasiva de la cultura de la clase obrera). La problemática etnográfica explorada por Willis ha sido analizada y elogiada por Marcus (1986, 245-262), quien considera saludable la renovación etnográfica propiciada por el enfoque de orientación neomarxista de Willis. 
En España, Feito (1990) ha publicado una investigación que sigue un diseño y una orientación teórica muy similares a los de Willis, constatando la divergencia de perspectivas entre el sistema escolar y los profesores, por un lado, y los estudiantes, por otro lado.

En una investigación de Everhart (1983) sobre los estudiantes de un instituto de bachillerato en los Estados Unidos encontramos una inquietud de orientación crítica que, hasta cierto punto, enlaza con las orientaciones del neomarxismo etnográfico británico. Everhart estudia la vida cotidiana de los alumnos en el centro educativo. La principal conclusión de este investigador, apoyada en autores como Habermas y R. Williams, es que los estudiantes desarrollan un interés técnico-instrumental hacia la institución y un interés práctico en el contexto de sus interacciones de grupo. Everhart contrapone el conocimiento reificado producido por la institución al conocimiento regenerativo creado por los alumnos en sus encuentros cotidianos. Desde esta perspectiva, la cultura de la adolescencia fomentada por la escuela, escribe Everhart $(1983,386)$, “...más que una cultura de liberación, se convierte en una fuerza productiva de reproducción cultural».

\section{LA INVESTIGACIÓN CUALITATIVA Y ETNOGRÁFICA DE CONTENIDO EDUCATIVO EN ESPAÑA}

No es nuestra intención describir detenidamente la investicación educativa española de enfoques cualitativo y etnográfico, sino tan sólo mencionar algunos títulos y caracterizar el momento actual en relación con esta temática. Uno de los primeros trabajos publicados es el de Knipmeyer et al. (1980), que incluye tres ensayos sobre antropología educativa, en los cuales se estudian las relaciones entre las escuelas y las comunidades locales, en tres ámbitos geográficos diferentes: el Polígono Cartuja de Granada; la comarca de Pallars Sobirá, en Lleida; y el barrio madrileño de La Celsa, cercano al Pozo del tío Raimundo.

En el ámbito de la sociología educativa, el trabajo ya mencionado de Feito (1990) es uno de los pocos que se han llevado a cabo con técnicas cualitativas, aunque algunos trabajos de Fernández Enguita han sido realizados con metodología de orientación cualitativa.

Los antropólogos han realizado varias investigaciones importantes, la mayor parte de ellas tesis doctorales no publicadas (cf. García Castaño y Pulido, 1994, 106-108, para una relación sobre estos trabajos) y, por tanto, de muy escasa difusión fuera de los circuitos académicos. Recientemente, ha sido publicada una de estas Tesis, que supone una importante contribución a la etnografía escolar en lengua española (Díaz de Rada, 1996).

Desde 1986, fecha del primer congreso sobre pensamiento de los profesores, se han publicado varios trabajos con enfoque etnográfico sobre temáticas docentes. A partir de esa fecha se evidencia el interés por unir investigación e innovación y por aproximar los enfoques cualitativo y cuantitativo (cf. Bartolomé, 1988). Entre los primeros estudios, figuran los de Montero (1986) y Zabalza (1988).

Entre las tesis doctorales, destacamos las siguientes: la de Arnaus (1993), que estudia la vida profesional y la práctica educativa de una maestra catalana de la 
segunda etapa de la EGB; la de García Jiménez (1991), que analiza la teoría y la práctica de la evaluación de un maestro de segunda etapa de EGB; la de Blanco, que consiste en un estudio de casos sobre un docente (cf. Blanco, 1994); la de González Sanmamed (1994), que trata del aprendizaje de la tarea de la enseñanza, mostrando la influencia de las prácticas de enseñanza en dos estudiantes de Magisterio; y la de González Monteagudo (1996), que combina las técnicas etnográficas clásicas con las historias de vida para ofrecer una interpretación de la vida profesional docente de dos maestros. Estos cinco trabajos están centrados en los profesores o estudiantes en formación. Sobre las prácticas de enseñanza y la formación inicial tratan los estudios de caso de Díaz Noguera (s.f.) y Pérez Ríos (s.f.), realizados en Andalucía y dirigidos por el profesor Pérez Gómez.

Los procesos de reforma de diferentes centros andaluces han sido analizados por Pérez Gómez (s.f.), Blanco (s.f.) y, sobre todo, por Martínez Rodríguez (1990 b; 1990 c; 1992), que ha estudiado en dos centros educativos el papel de los alumnos en los procesos de innovación y cambio curricular. Por otra parte, en Cataluña, Sancho et al. (1993) han investigado los procesos de innovación en tres contextos escolares diferentes.

En una reciente revisión de las investigaciones cualitativas sobre temática educativa realizadas en España y publicadas en revistas entre los años 1987 y 1993, Colás (1994) identifica una veintena de estudios, cuyo comentario muestra la riqueza de planteamientos metodológicos y de temáticas que han aparecido en España a partir de finales de los años ochenta. Es indudable que en los próximos años vamos a presenciar un fuerte auge de los planteamientos etnográficos y cualitativos.

Esperamos con este texto, y con la recopilación bibliográfica que lo acompaña, poder contribuir a la difusión de la Antropología y etnografía educativas, cuyas aportaciones a la teoría, la investigación, la docencia y la intervención en los ámbitos educativos están todavía por descubrir.

\section{BiBLIOGRAFÍA}

AGUiRRE, A. (Ed.) (1995): Etnografía. Metodología cualitativa en la investigación sociocultural. Marcombo y Boixareu Universitaria, Barcelona.

ARNAUS, R. (1993): Vida professional i acció pedagògica. A la recerca de la comprensió d'una mestra. Un estudi de cas. Dpto. de Didáctica y Organización Escolar, División de Ciencias de la Educación, Univ. de Barcelona, Tesis Doctoral inédita.

AtKinson, P. \& Delamont, S. (1990): Writing about teachers: How british and american ethnographic texts describe teachers and teaching. Teaching \& Teacher Education, vol. 6, no 2, pp. 111-125.

AtKInson, P. et al. (1988): Qualitative Research traditions: A british response to Jacob. Review of Educational Research, vol. 58, no 2, pp. 231-250.

AzNar, P. y Requejo, A. (1993): La investigación etnográfica en la educatión no formal. En NÚNEEz CUBERo, L. (Ed.): Metodologias de la investigación en la Educación no Formal. aportaciones teóricas. GIT-PreuSpínola, Sevilla, pp. 135-186.

Bartolomé, M. (1988): Nuevas tendencias en los diseños de investigación en España. En Dendaluce, I. (Coord.): Aspectos metodológicos de la investigación educativa. Narcea y II Congreso Mundial Vasco, Madrid, pp. 102-120. 
Blanco, N. (1994): Contexto institucional y práctica docente. Estudio de un caso. Revista de Educación, no 303, pp. 329-350.

Bogdan, R. C. \& BiKLEN, S. K. (1982): Qualitative Research for education.An introduction to theory and methods. Allyn \& Bacon, Boston.

Bouché, J. H. (1994): Etnografía, etnología y antropología (Un enfoque pedagógico). En López Barajas, E y Montoya, J. M. (Eds.): La investigación etnogrăfica. Fundamentos y técnicas. U.N.E.D., Madrid, pp. 69-85.

Bouché, J. H. et al. (1995): La Antropología de la Educación como disciplina: Proyecto de diseño. Teoría de la Educación, no 7, pp. 95-114.

CARSPECKEN, P. F. \& APPLE, M. W. (1992): Critical Qualitative Research: Theory, methodology, and practice. En LECOMPTE, M. D. et al. (Eds.): The Handbook of Qualitative Research in education. Academic Press, San Diego, pp. 507-554.

CHarlot, B. (1989): L'ethnographie de l'école dans les travaux britanniques. Practiques de Formation, no 18 , pp. 87-106.

CuIfFord, J. (1986): Introducción: verdades parciales. En ClifFord, J. y MARCus, G. E. (Eds.): Retóricas de la Antropología. Júcar, Madrid, pp. 25-60.

Cufford, J. y Marcus, G. E. (Eds.): Retóricas de la Antropología. Júcar, Madrid.

Colom, A. J. y Mèulch, J. C. (1994): Antropología y educación. Notas sobre una difícil relación conceptual. Teoría de la Educación, no 6, pp. 11-21.

Delamont, S. (1976): La interacción didáctica. Cincel, Madrid.

Delamong, S. \& Galton, M. (1986): Inside the secondary classroom. Routledge \& Kegan Paul, London.

Delamont, S. y Hamilton, D. (1978): Investigación en el aula: Una crítica y un nuevo planteamiento. En StUBBS, M. y DeLAMONT, S. (Eds.): Las relaciones profesor-alumno, OikosTau, Barcelona, pp. 15-33.

DÍAz DE RADA, A. (1996): Los primeros de la clase y los últimos románticos. Una etnografía para la critica de la visión instrumental de la enseñanza. Siglo XXI, Madrid.

Díaz Noguera, $M^{a}$ D. (s.f.): La realidad vivida por Luis: las prácticas de un futuro profesor de Educación Física. En ANGUITA, R. et al. (s.f.): Las prácticas de enseñanza. La vivencia de los futuros enseñantes. Estudios de casos. Sevilla, Documento policopiado, pp. 39-59.

Díaz Viana, L. (1991): Prólogo a la edición española. En Cuifford, J. y Marcus, G. E. (Eds.) (1986): Retóricas de la Antropología. Júcar, Madrid, pp. 9-19.

EDDY, E. M. (1985): Theory, research, and application in educational Anthropology. Antbropology \& Education Quarterly, vol. 16, $\mathrm{n}^{\mathrm{O}} 2$, pp. 83-104.

Estebaranz, A. y Mingorance, P. (1995): Factores socioculturales que influyen en la educación: La aportación del modelo etnográfico. Bordón, vol. 47, $\mathrm{n}^{\circ}$ 4, pp. 417-425.

Esteve, J. M. et all. (1993): Interaccionismo simbólico. En NúÑ̃z Cubero, L. (Ed.): Metodologías de la investigación en la Educación no Formal. Aportaciones teóricas. GIT-PreuSpínola, Sevilla, 61-99.

EverHart, R. B. (1983): Leer, escribir y resistir. En Velasco, H. M. et al. (Eds.) (1993): Lecturas de Antropología para educadores. Trotta, Madrid, pp. 355-388.

FEITO, R. (1990): Nacidos para perder. Un análisis sociológico del rechazo y del abandono escolares. CIDE-MEC, Madrid.

FERNÁNDEZ ENGUITA, M. (1990): La escuela a examen. Eudema, Madrid.

García Castaño, J. y Pulddo, R. A. (1994): Antropología de la Educación. Eudema, Madrid.

GARCíA JimÉNEZ, E. (1991): Una teroía práctica sobre la evaluación. Un estudio etnográfico, MIDO, Sevilla.

GEERTZ, C. (1973): La interpretación de las culturas. Gedisa, Barcelona, 1987.

GEERTZ, C. (1988): El antropólogo como autor. Paidós, Barcelona, 1989. 
GeERTZ, C. et al. (Eds.) (1992): El surgimiento de la Antropología posmoderna. Gedisa, Barcelona, 1993.

Giroux, H. A. (1988): Los profesores como intelectuales. Hacia una pedagogía crítica del aprendizaje. Paidós/MEC, Barcelona/Madrid, 1990.

GonzÁlez Monteagudo, J. (1994): El estudio de los profesores desde la Antropología Educativa. En VV.AA.: III Simposi Internacional de Filosofía de l'Educació. Vol. 2: Comunicacions. Univ. Autónoma y Univ. Ramón Llull, pp. 71-76.

GonzÁlez MonTEagudo, J. (1996): Vida cotidiana y profesión docente: teoría y práctica educativas centradas en bistorias de vida. Un enfoque etnográfico. Univ. de Sevilla. Dpto. de Teoría e Historia de la Educación y Pedagogía Social. Tesis Doctoral inédita.

GONZÁLEZ SANMAMED, M. (1994): Aprender a enseñar: mitos y realidades. Univ. de La Coruña, La Coruña.

HAMmERSLEY, M. (Ed.) (1984 a): The ethnography of schooling: Methodological issues. Nafferton Books, Driffield.

HAMmERSLeY, M. (1984 b): Introduction: Reflexivity and naturalism in ethnography. En HAMmERSLEY, M. (Ed.): The ethnography of schooling: Methodological issues. Nafferton Books, Driffield, pp. 1-18.

HAMmERSLEY, M. (1990): Classroom etbnography. Empirical and methodologial essays. Open University Press. Milton Keynes, Philadelphia.

HAMMERSLEY, M. (1992): Some reflections on ethnography and validity. International Journal of Qualitative Studes in education, vol. 5, no 3, 195-203.

HAMmERSLEY, M. y ATKINSON, P. (1983): Etnografia. Métodos de investigación. Paidós, Barcelona, 1994.

Hargreaves, A. (1992): El tiempo y el espacio en el trabajo del profesor. Revistas de Educación, n⿳⺈ 298, pp. 31-53.

HARRINGTON, C. C. (1982): Anthropology. En Encyclopedy of educational research. Free Press, New York, pp. 132-137.

HARRIS, M. (1968): El desarrollo de la teoria antropológica. Una historia de las teorias de la cultura. Siglo XXI, Madrid, 1987.

HaRRIS, M. (1983): Antropología cultural. Alianza, Madrid, 1990.

Hymes, D. (1982): ¿Qué es la etnografía? En Velasco, H. M. et al. (eds.) (1993): Lecturas de Antropologia para educadores. Trotta, Madrid.

JACKSON, P. W. (1968): La vida en las aulas. Marova, Madrid, 1975 (nueva ed. en Morata, Madrid, 1991).

JANER, G. y COLOM, A. J. (1995): El modelo cultural en la construcción de la Antropología de la Educación. En Noguera, J. (Ed.): Cuestiones de Antropología de la Educación. Ceac, Barcelona, pp. 63-94.

JimÉNEZ NúÑEZ, A. (1979): Antropología cultural. Una introducción a la ciencia de la educación. MEC, Madrid.

Kimbali, S. T. (1955): The method of natural history and educational research. En SPINDLER, G. \& SPINDLER, L (Eds.) (1987): Interpretative etbnography of education: at bome and abroad. Lawrence Erlbaum Associates, Hillsdal, N. J., pp. 11-16.

KNIPMEYER, M. et al. (1980): Escuelas, pueblos y barrios: tres ensayos de antropología educativa. Akal, Madrid.

LAPASSADE, G. (1991): L'Ethnosociologie. Les sources anglo-saxons. Méridiens Klincksieck.

MALINOWSKI, B. (1922): Introducción: objeto, método y finalidad de esta investigación. En Velasco, H. M. et al. (Eds.) (1993): Lecturas de Antropología para educadores. Trotta, Madrid, pp. 21-42.

Marcus, G. E. (1986): Problemas de la etnografía contemporánea en el mundo moderno. En Clifford, J. y Marcus, G. E. (Eds.): Retóricas de la Antropología. Júcar, Madrid, pp. 235-268. 
Marcus, G. E. (1994): What come (just) after "Post»? The case of ethnography. En DenzIN, N. K. \& LiNCOLN, Y. S. (Eds.): Handbood of Qualitative research. Sage, London, pp. 563-574.

Martínez Rodríguez, J. B. (Ed.) (1990 a): Hacia un enfoque interpretativo de la enseñanza. Univ. de Granada, Granada.

Martínez Rodríguez, J. B. (1990 b): Informe sobre el Ciclo Superior en un colegio público. En Martínez Rodríguez, J. B. (Ed.): Hacia un enfoque interpretativo de la enseñanza. Univ. de Granada, Granada, pp. 145-230.

MarTínez Rodríguez, J. B. (1990 c): Algunas ideas para trabajar en didáctica aplicando la metodología etnográfica. En Martínez Rodríguez, J. B. (Ed.): Hacia un enfoque interpetativo de la eneñanza. Univ. de Granada, Granada, pp. 9-50.

Martínez Rodríguez, J. B. (1992): El alumnado y la reconstrucción del curriculum en la Reforma. Univ. de Granada, Granada.

MCLAREN, P. (1986): La escuela como un performance ritual. Hacia una economía politica de los simbolos y gestos educativos. Siglo XXI, México, 1995.

MeAd, M. (1930): Educación y cultura. Paidós, Buenos Aires, 1962.

MEAD, M. (1972): Experiencias personales y científicas de una antropóloga. Paidós, Barcelona, 1994.

MÈLICH, J. C. (1996): Antropología simbólica y acción educativa. Paidós, Barcelona.

Montero, L. M a (1986): Pensamiento de los profesores, investigación cualitativa y formación del profesorado. En Villar ANGuLo, L. M. (Ed.): Pensamiento de los profesores y toma de decisiones. Univ. de Sevilla, Sevilla, pp. 333-341.

OGBU, J. U. (1974): The Next generation. An etbnography of education in an urban neighborbood. Academic Press, New York.

OgBu, J. U. (1981): Etnografía escolar. Una aproximación a nivel múltiple. En Velasco, H. M. et al. (Eds.) (1993): Lecturas de Antropología para educadores. Trotta, Madrid, pp. $145-174$

OGBu, J. U. (1989): Antropología de la Ecucación. En Husen, T. y Postlethwarte, T. N. (Dirs.) (1989-1993): Enciclopedia Internacional de Educación. Vicens-Vives/MEC, Barcelona/ Madrid, vol. 1, pp. 291-313.

Pérez Gómez, A. (s.f.): La Reforma desde dentro. Estudio múltiple de casos. Documentos de Evaluación del Ciclo Superior de EGB en Andalucía, no 8. Consejería de Educación y Ciencia, Junta de Andalucía, Sevilla.

Pérez Ríos, J. (s.f.): Estudio de caso sobre las prácticas de enseñanza en la formación inicial en E.U. de Formación del Profesorado de Cádiz ( $1^{\mathrm{a}}$ y $2^{\mathrm{a}}$ fases). En ANGuiTA, R. et al. (s.f.): Las prácticas de enseñanza. La vivencia de los futuros enseñantes. Estudios de casos. Documento policopiado, pp. 218-295.

QuANTZ, R. A. (1992): On critical ethnography (with some postmodern considerations). En LeCompte, M. D. et al. (Eds.): The Handbook of Qualitative Research in education. Academic Press, San Diego, pp. 447-506.

Rrvas, J. I. (1990): El aula como unidad de socialización: los rituales de aprendizaje. Educación y sociedad, $\mathrm{n}^{\mathrm{Q}}$ 7, pp. 73-90.

ROMAN, L. G. (1992): The political significance of other ways of narrating ethnography: a feminist materiaslist approach. En LECOMPTE, M. D. et al. (Eds.): The Handbook of Qualitative Research in education. Academic Press, San Diego, pp. 555-594.

RomÁn, L. G. \& APpLE, M. W. (1991): ¿Es el naturalismo un alejamiento del positivismo? Los enfoques materialista y feminista de la subjetividad en la investigación etnográfica. Educación y sociedad, no 9, pp. 59-90.

SÁNCHEZ-CANDAmio, M. (1995): La etnografía en psicología social. Revista de Psicología Social Aplicada, vol. 5, $\mathrm{n}^{-}$1/2, pp. 27-40. 
SANCHO, J. Ma et al. (1993): Aprendiendo de las innovaciones en los centros. La perspectiva interpretativa aplicada a tres estudios de casos. CIDE-MEC, Madrid.

SANDAY, P. R. (1983): The ethnographic paradigm(s). En VAN MAANEN, J. (Ed.): Qualitative methodology. Sauge, London, pp. 19-36.

SCHENSUL, J. J. (1985): Cultural maintenance and cultural transformation: Educational Anthropology in the eighties. Antrhopology \& Education Quarterly, vol. 16, $\mathrm{n}^{\circ} 1$, pp. 63-68.

SCHWANDT, T. (1994): Constructivist, interpretivist approaches to human inquiry. En DenzIN, N. K. \& Lincoln, Y. S. (Eds.): Handbook of Qualitative Research in education. Sage, London, pp. 118-137.

SiRota, R. (1987): Approches ethnographiques en sociologie de l'éducation: l'école et la communauté, l'établissement scolaires, la classe. Revue Française de Pédagogie, $\mathrm{n}^{\circ}$ 80, pp. 69-89.

SMTth, L. M. \& GEOFFREY, W. (1968): The complexities of an urban classroom. Holt, Rinehart $\&$ Winston, New York.

SPINDLER, G. (Ed.) (1982): Doing the ethnography of schooling. Educational Anthropology in action. Holt, Rinehart \& Winston, New York.

Spindler,G. (1987): La transmisión de la cultura. En Velasco, H. M. et al. (Eds.) 1993): Lecturas de Antropología para educadores. Trotta, Madrid, 205-242.

SPINDLER, G. \& SPINDLER, L. (Eds.) (1987 a): Interpretative etbnography of education: at home and abroad. Lawrence Erlbaum Associates, Hillsdal, N. J.

SPINDLER, G. \& SPINDLER, L. (1987 b): Teaching and learning how to do the ethnography of education. En SPINDLER, G. \& SPINDLER, L. (Eds.): Interpretive ethnography of education: at home and abroad. Lawrence Erlbaum Associates, Hillsdal, N. J.,pp. 17-36.

SPINDLER, G. \& SPINDLER, L. (1992): Cultural processa and ethnography: an anthropologicalperspective. En LECOMPTE, M. D. et al. (Eds.): The Handbook of Qualitative Research in education. Academic Press, San Diego, pp. 53-92.

Spradley, J. P. (1979): The ethnographic interview. Holt, Rinehart \& Winston, New York.

STENHOUSE, L. (1981): Investigación y desarrollo del curriculum. Morata, Madrid, 1984.

STOcking, G. W. Jr. (1983): La magia del etnógrafo. El trabajo de campo en Antropología británica desde Tylor a Malinowski. En Velasco, H. M. et al. (Eds.) (1993): Lecturas de Antropología para educadores. Trotta, Madrid,pp. 43-93.

TORRES, J. (1994): Globalización e interdisciplinariedad. El curriculum integrado. Morata, Madrid.

TYleR, S. A. (1986): Etnografía postmoderna: Desde el documento de lo oculto a lo oculto del documento. En Clifford, J. y Marcus, G. E. (Eds.): Retóricas de la Antropología. Júcar, Madrid, pp. 183-204.

VÁzQuez, A. y MARTíNEZ, I. (1996): La socialización en la escuela. Una perspectiva etnográfica. Paidós, Barcelona.

WILCOX, K. (1982 a): La etnografía como una metodología y su aplicación al estudoi de la escuela: una revisión. En Velasco, H. M. et al. (Eds.) (1993): Lecturas de Antropología para educadores. Trotta, Madrid, pp. 95-126.

WILCOX, K. (1982 b): Differential socialization in the classroom: implications for equal opportunity. En SPINDLER, G. (Ed.): Doing the ethnography of schooling. Educational Anthropology in action. Holt, Rinehart \& Winston, New York, pp. 268-309.

WILLIS, P. (1977): Aprendiendo a trabajar. Cómo los chicos de la clase obrera consiguen trabajos de clase obrera. Akal, Madrid, 1988.

Willis, P. (1981): Producción Cultural no es lo mismo que Reproducción Cultural, que a su vez no es lo mismo que Reproducción Social, que tampoco es lo mismo que 
Reproducción. En Velasco, H. M. et al. (Eds.) (1993): Lecturas de Antropología para educadores. Trotta, Madrid, pp. 431-461.

Wolcotr, H. F. (1974): El maestro como enemigo. En Velasco, H. M. et al. (Eds.) (1993): Lecturas de Antropologia para eduadores. Trotta, Madrid, pp. 243-258.

WolcotT, H. F. (1982): Mirrors, models, and monitors: Educator adaptations of the ethnographic innovation. En SPINDLER, G. (Ed.): Doing the ethnography of schooling. Educational Antbropology in action. Holt, Rinehart \& Winston, New York, pp. 68-95.

WolcotT, H. F. (1985): Sobre la intención etnográfica. En Velasco, H. M. et al. (Eds.) (1993): Lecturas de Antropología para educadores. Trotta, Madrid, pp. 127-144.

WolcotT, H. F. (1992): Posturing in Qualitative Research. En LeComPte, M. D. et al. (Eds.): The Handbook of Qualitative Research in education. Academic Press, San Diego, pp. 3-52.

Wolct, H. F. (1994): Transforming qualitative data. Description, analysis, and interpretation. Sage, New York.

Woods, P. (1986): La escuela por dentro. La etnografía en la investigación educativa. Paidós/MEC, Barcelona/Madrid, 1989.

Woods, P. (1990 a): Reflexiones sur quelques aspects de l'ethnographie interactoiniste de l'école (Entretien entre P. Woods, et P. Boumard). Pratiques de Formation, $\mathrm{n}^{\circ}$ 20,pp. 169-176.

Woods, P. (1990 b): L'ethnographie de lécole. Armand Colin, Paris.

Woods, P. (1990 c): Teachers skills and strategies. Falmer Press, London.

Woods, P. (1990 d): Educational ethnography in Britain. En SHERman, R. R. \& WebB, R. B. (Eds.): Qualitative research in education: Focus and methods. Falmer Press, London, pp. 90-109.

Woods, P. (1992): Symbolic interaccionism: theory and method. En LECOMPTE, M. D. et al. (Eds.): The Handbook of Qualitative Research in education. Academic Press, San Diego, pp. 337-404.

Woods, P. (1994): Teachers under siege: Resistance and appropiation in English Primary Schools. Antbropology \& Education Quarterly, vol. 25, nำ 3, pp. 250-265.

Woods, P. y Hammersley, M. (Comps.) (1994): Género, cultura y etnia en la escuela. Informes etnográficos. Paidós/MEC, Barcelona/Medrid, 1995.

ZABALZA, M. A. (1988): Los diarios de clase como documentos para estudiar cualitativamente los dilemas prácticos de los profesores. Univ. de Santiago, Santiago.

ZAHARLICK, A. (1992): Ethnography in Anthropology and its value for education. Theory into Practice, vol. 31, nำ 2, pp. 116-125. 